\title{
A model finding a new Richardson potential with different scales for confinement and asymptotic freedom, by fitting the properties of $\Delta^{++}$and $\Omega^{-}$.
}

\author{
Manjari Bagchi ${ }^{1,3, *} \S$, Mira Dey ${ }^{1,3, *}$, Sukanta Daw ${ }^{1,3}$ and Jishnu Dey ${ }^{2,3, *, \dagger}$.
}

November 13, 2018

\begin{abstract}
Phenomenological Richardson potential has built in asymptotic freedom (AF in short) and confinement, with only one parameter $\Lambda$ in the potential. But it is known that the scales of $\mathrm{AF}$ and confinement are not the same. In the present work a relativistic mean field calculation for baryons is tried out with two parameters $\Lambda$ and $\Lambda^{\prime}$ for $\mathrm{AF}$ and confinement respectively .

To test the two parameter potential we calculate the energies and the magnetic moments, of the triple $\mathrm{u}$ - quark system $\left(\Delta^{++}\right)$and the triple $\mathrm{s}$ - quark system $\left(\Omega^{-}\right)$and found good values for $\Lambda=100 \mathrm{MeV}$ and $\Lambda^{\prime}=350 \mathrm{MeV}$. So we believe that the modified Richardson potential should have AF scale $\Lambda=100 \mathrm{MeV}$ and the confinement scale $\Lambda^{\prime}=350 \mathrm{MeV}$.
\end{abstract}

Keywords: Hartree-Fock -Richardson Potential- magnetic moment - dense matter.

${ }^{1}$ Dept. of Physics, Presidency College, 86/1, College Street, Kolkata 700 073, India

2 UGC Research Professor, Dept. of Physics, Maulana Azad College, 8 Rafi Ahmed Kidwai Road, Kolkata 700 013, India

${ }^{3}$ Visitor (2003), IUCAA, Pune, India

$\dagger$ Project : Changing Interface of Nuclear, Particle and Astrophysics, email : kamal1@vsnl.com.

* Work supported in part by DST grant no. SP/S2/K-03/2001, Govt. of India.

$\S$ email : mnj2003@vsnl.net. 


\section{Introduction}

't Hooft suggested that the inverse of the number of colors $N_{c}$ could be used as an expansion parameter in the otherwise parameter free QCD theory [1]. By the end of 90's, properties of large $N_{c}$ baryons have been extensively studied by algebraic methods for spin and isospin symmetry. It has become possible to make a unified view on the various effective theories such as the Skyrme model, the non-relativistic quark model and the chiral bag model [2]. Witten [3] suggested that for large $N_{c}$ baryons a mean field description could be obtained using a phenomenological interquark potential tested in the meson sector ${ }^{1}$

Indeed self consistent baryon mass calculation is feasible with success in the mean field level [4] using the Richardson potential [5] as an interquark interaction. Richardson potential takes care of the two features of the qq force, asymptotic freedom and confinement as given below :

$$
V\left(r_{12}\right)=-\frac{N_{c}^{2}-1}{2 N_{c}} \frac{6 \pi}{33-2 N_{f}}\left[\Lambda^{2} r_{12}-f\left(\Lambda r_{12}\right)\right]
$$

where $-\frac{N_{c}^{2}-1}{2 N_{c}}$ is the color contribution, $N_{f}$ is the number of flavors, taken to be three, and

$$
f(t)=1-4 \int_{1}^{\infty} \frac{d q}{q} \frac{\exp (-q t)}{\left[\ln \left(q^{2}-1\right)\right]^{2}+\pi^{2}}
$$

$\Lambda$ is a parameter whose value was originally chosen as $400 \mathrm{MeV}$ as for small $q^{2}$ the potential reduces to a linear confinement, and the linear confinement string tension from lattice calculation is about that value. However, the asymptotic freedom part also has the same $\Lambda$. In other words, both the confinement and asymptotic freedom scales are chosen to be the same in the potential equation (1) even though it is known that the asymptotic freedom scale should be around $100 \mathrm{MeV}$.

Same potential has been included in the relativistic HF calculation of strange quark matter to form compact stars. These stars are more compact than the conventional neutron stars and fit into the Bodmer-Witten hypothesis for the existence of strange quark matter. They lead to systems more compact than those derived assuming the bag model type of quark confinement $[6,7,8]$ and leads to various observable phenomenon $[9,10,11,12,13,14,15,16,17,18]$. But $\Lambda$ parameter had to be within $100-200 \mathrm{MeV}$. This is consistent with asymptotic freedom scale but for confinement scale, it is low.

Since the scales of these two phenomena, need not be the same as in the original potential., we incorporate them explicitly as $\Lambda^{\prime}$ and $\Lambda$ :

$$
V\left(r_{12}\right)=-\frac{N_{c}^{2}-1}{2 N_{c}} \frac{6 \pi}{33-2 N_{f}}\left[\Lambda^{\prime 2} r_{12}-f\left(\Lambda r_{12}\right)\right]
$$

\footnotetext{
${ }^{1}$ We agree with the referee that a relativistic Faddeev calculation would be more appropriate for a 3 quark system but do not attempt that. This is because our goal is to find an effective interaction from finite system for use in strange star calculations.
} 
$\Lambda^{\prime}$ corresponds to the confinement part and $\Lambda$ corresponds to the asymptotic freedom part.

Recently the magnetic moment of $\Delta^{++}$was derived from experimental data [19] and it was shown in [20] that many models of hadrons cannot fit its value satisfactorily. We re-visit the relativistic mean field calculation [4] with two parameter Richardson potential (3). Elsewhere the star calculation is repeated with the modified potential ([21]).

In this work an extensive search is performed to find the magnetic moment and mass of the $\Delta^{++}$and $\Omega^{-}$. It is found that for the confinement part a $\Lambda^{\prime}$ of about $340-350 \mathrm{MeV}$ and for asymptotic part a $\Lambda$ of $100 \mathrm{MeV}$ reproduces both the magnetic moment and the energy satisfactorily.

This relatively small value of asymptotic freedom parameter $(\Lambda)$ is in agreement with Shifman et al [22] (70 MeV - $100 \mathrm{MeV})$.

Interestingly, in a recent paper, Radzhabov and Volkov [23] proposed that $\Lambda^{\prime}$ should be 340 $\mathrm{MeV}$.

\section{Details of calculation}

Assuming the quarks occupy the same orbital, the mean field $\omega_{a v}(r)$ is given by

$$
\omega_{a v}(\vec{r})=-\frac{N_{c}^{2}-1}{2 N_{c}} \int \phi_{j m}^{\dagger}\left(\vec{r}^{\prime}\right) V\left(\vec{r}-\vec{r}^{\prime}\right) \phi_{j m}\left(\vec{r}^{\prime}\right) \overrightarrow{d r}^{\prime}
$$

where $-\frac{N_{c}^{2}-1}{2 N_{c}}$ is the color contribution in the color singlet state, the Fock term. The color factor for the Hartree term is zero. The energy $E_{H F}$ is given by

$$
E_{H F}=N_{c}\left(\epsilon-\frac{1}{2}<\phi_{j m}\left|\omega_{a v}\right| \phi_{j m}>\right)
$$

where $\epsilon$ is the single particle energy obtained by solving

$$
\left[\vec{\alpha} \cdot \vec{p}+\beta m+\omega_{a v}(r)\right] \phi_{j m}(r)=\epsilon \phi_{j m}(r)
$$

where $\alpha$ 's and $\beta$ are the usual Dirac matrices. For quarks in the lowest $\left(1 s_{1 / 2}\right)$ orbital, one may write

$$
\phi_{1 s_{1 / 2}}(r)=\left[\frac{1}{4 \pi}\right]^{\frac{1}{2}}\left(\begin{array}{c}
i G(\vec{r}) \chi_{m} \\
\vec{\sigma} \cdot \hat{r} F(\vec{r}) \chi_{m}
\end{array}\right)
$$

where $\chi_{m}$ is the Pauli spinor and the eqn(5) yields the system of coupled differential equations:

$$
\begin{gathered}
\frac{d G}{d r}-\left(m-\omega_{a v}+\epsilon\right) F=0 \\
\frac{d F}{d r}+\left(\frac{2}{r}\right) F+\left(\epsilon-\omega_{a v}-m\right) G=0
\end{gathered}
$$


The above equations are solved self-consistently by iteration since $\omega_{a v}$ depends on Dirac large and small components $G(r)$ and $F(r)$.

In eqns (8) and (9), the self consistent single particle confining potential is a vector one, leading to instability in the solution. The same problem was encountered by Crater and Van Alstine [24] who suggested a prescription of taking a half-vector half-scalar form for the linear part. This choice also leads to a cancellation of spin-orbit effects at long range. So we add the confining part of the two-body potential equally to the energy and mass ( $1 / 2$ vector, $1 / 2$ scalar potential).

The vector and scaalar potentials are respectively:

$$
\begin{gathered}
V_{v e c}\left(r_{12}\right)=-\frac{N_{c}^{2}-1}{2 N_{c}} \frac{6 \pi}{33-2 N_{f}}\left[\frac{\Lambda^{\prime 2} r_{12}}{2}-f\left(\Lambda r_{12}\right)\right] \\
V_{\text {scalar }}\left(r_{12}\right)=-\frac{N_{c}^{2}-1}{2 N_{c}} \frac{6 \pi}{33-2 N_{f}} \frac{\Lambda^{\prime 2} r_{12}}{2}
\end{gathered}
$$

The vector potential $V_{v e c}\left(r_{12}\right)$ is used in the expression of $\omega_{a v}(\vec{r})$ [equation 4] and the scalar potential $V_{\text {scalar }}\left(r_{12}\right)$ is added with the mass terms in the coupled differential equations.

The magnetic moment $\mu$ is given by (see [25]):-

$$
\mu=-e_{B} \frac{2}{3} \int_{0}^{\infty} G(r) F(r) r^{3} d r
$$

where $e_{B}$ is the charge of the baryon.

It is convenient to express the Dirac components $\mathrm{G}(\mathrm{r})$ and $\mathrm{F}(\mathrm{r})$ as sum of oscillators :

$$
\begin{aligned}
G(r) & =\sum_{n} C_{n} R_{n 0} \\
F(r) & =\sum_{m} D_{m} R_{m 1}
\end{aligned}
$$

where C's and D's are coefficients. The expansion reduces the differential equation to an eigenvalue problem. The solution is to be found self consistently by diagonalizing the matrix and putting back the coefficients till convergence is reached. In general $R_{n l}(r)$ is given by :

$$
R_{n l}(r)=\sqrt{\frac{2 n !}{\Gamma\left(n+l+\frac{3}{2}\right)}} r^{l} \exp \left(-\frac{1}{2} r^{2}\right) L_{n}^{l+\frac{1}{2}}\left(r^{2}\right)
$$

where $L_{n}^{l+\frac{1}{2}}\left(r^{2}\right)$ are Associate Laguerre polynomials.

In the calculation, $\mathrm{r}$ is replaced by $\mathrm{r} / \mathrm{b}$ in the expression of $L_{n}^{l+\frac{1}{2}}\left(r^{2}\right)$ where $\mathrm{b}$ is the oscillator length which need not to be the same for $\mathrm{G}(\mathrm{r})$ and $\mathrm{F}(\mathrm{r})$ and they are denoted by $b$ and $b^{\prime}$ respectively. 
Starting with a trial set of C's and D's , $\omega_{a v}$ is calculated, a matrix is constructed and diagonalized. The new coefficients $C_{n}$ and $D_{n}$ can be read off from the eigenvector with the corresponding eigenvalue $\epsilon$. The wave functions are put back in the equations and the process is continued till self consistency is reached.

But the problem is that the RHF solutions violate translational invariance, since they are formed by single-particle wave functions derived by an average potential $\omega_{a v}$. As a consequence, the centre-of-mass momentum is not well-defined in RHF solutions and this entails a spurious contribution from the centre-of-mass $[\mathrm{CM}]$ kinetic energy to the total energy. Since the relative importance of this effect increases as the number of particles decreases, it is important that it should be corrected for systems formed of few particles. This can be done by extending to the RHF equations the Peierls-Yoccoz procedure of nuclear physics. The spurious contribution is denoted by $T_{C M}$ and the baryon mass $[\mathrm{M}]$ has to be compared with the difference $E_{H F}-T_{C M}$. Here this spurious contribution has also been calculated and subtracted from $E_{H F}$ to estimate the correct energy of the baryon.

We have also estimated the values of r.m.s. radius $r_{a v}$ and checked if the wave functions are normalized or not. The normalization factor is denoted by $\mathrm{N}$.

$r_{a v}$ is given by:

$$
r_{a v}=\sqrt{\int_{0}^{r_{\max }}\left(G(r)^{2}+F(r)^{2}\right) r^{4} d r}
$$

$\mathrm{N}$ is given by:

$$
N=\int_{0}^{r_{\max }}\left(G(r)^{2}+F(r)^{2}\right) r^{2} d r
$$

where $r_{\max }$ is the upper limit of integration and is taken as $5.0 \mathrm{fm}$ to make $\mathrm{N} \sim 1$.

\section{Results for $\Delta^{++}$}

We start with the simplest system, the totally symmetric spin and isospin triple u-quark state. Here $m_{u}$ is taken as $4 \mathrm{MeV}$. We checked the convergence in choosing the number of the oscillators. In figure [1] we see that the change in $E_{H F}$ is $32 \mathrm{MeV}$ when we increase the matrix dimension from 5 X 5 to $7 \mathrm{X} 7$, but only $16 \mathrm{MeV}$ from $7 \mathrm{X} 7$ to $9 \mathrm{X} 9$. It is interesting to note that the magnetic moment remains almost same when we increase the dimension from $7 \mathrm{X} 7$ to $9 \mathrm{X} 9$. In table 1 , it is shown that $E_{H F}$ is almost independent of oscillator parameter $b$ and $b^{\prime}$ and the mass after $\mathrm{CM}$ correction is $1171 \mathrm{MeV}$.

Now, we vary $\Lambda$ and $\Lambda^{\prime}$ independently. We give details of $\Lambda$ and $\Lambda^{\prime}$ variational results in table 2 and table 3 for matrix dimension 7 X 7 as an example. We find that $E_{H F}$ varies widely with $\Lambda^{\prime}$ as expected since $\Lambda^{\prime}$ is the confining parameter. With $\Lambda^{\prime}=350 \mathrm{MeV}$ and $\Lambda=100 \mathrm{MeV}$ ( table [2] ) $\Delta$ mass $=1250 \mathrm{MeV}$ and its magnetic moment 5.77 magneton. Our final result is given in table [4] for $9 \mathrm{X} 9$ matrix. We get the $\Delta$ mass to be $1224 \mathrm{MeV}$ and its magnetic moment $\mu=6.15$ magneton. 
Table 1: Variation of Hartree Fock energy, centre of mass kinetic energy, mass and magnetic moment of $\Delta^{++}$with oscillator parameter $b$ where $\Lambda=\Lambda^{\prime}=350 \mathrm{MeV}$; for 9 X 9 matrix.

\begin{tabular}{|c|c|c|c|c|c|c|c|}
\hline$b^{\prime}$ & $b$ & $E_{H F}(\mathrm{MeV})$ & $T_{C M}(\mathrm{MeV})$ & $\mathrm{M}(\mathrm{MeV})$ & $\frac{\mu}{\mu_{0}}$ & $r_{a v}(\mathrm{fm})$ & $N$ \\
\hline 0.60 & 0.78 & 1310 & 139 & 1171 & 6.75 & 1.30 & 0.988152 \\
\hline 0.60 & 0.80 & 1307 & 138 & 1169 & 6.69 & 1.31 & 0.990868 \\
\hline 0.60 & 0.82 & 1303 & 138 & 1165 & 6.56 & 1.32 & 0.995152 \\
\hline 0.60 & 0.84 & 1301 & 130 & 1171 & 6.42 & 1.31 & 0.998549 \\
\hline 0.60 & 0.86 & 1299 & 130 & 1169 & 6.30 & 1.31 & 1.000855 \\
\hline 0.60 & 0.88 & 1298 & 127 & 1171 & 6.23 & 1.30 & 1.002296 \\
\hline
\end{tabular}

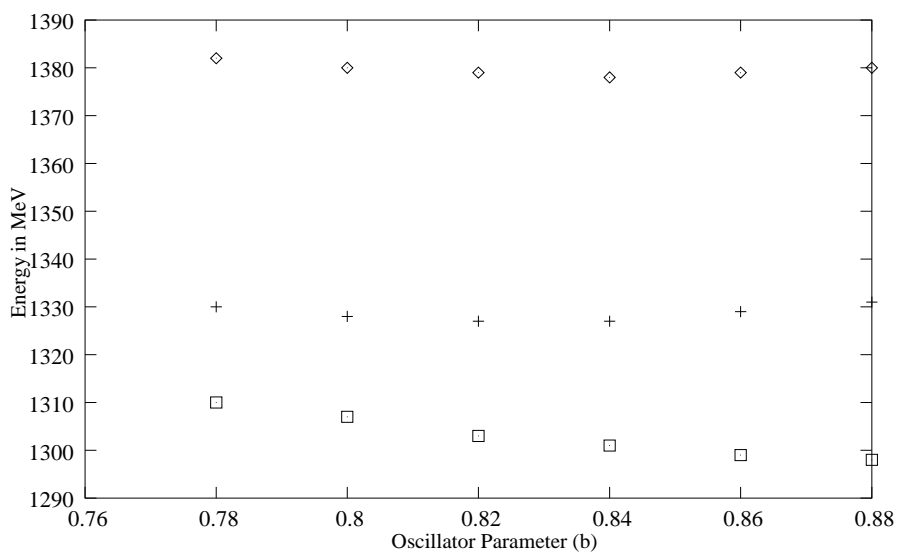

Figure 1: Variation of Hartree-Fock energy $E_{H F}$ with Oscillator Parameter $b$ for 5 by 5,7 by 7 and 9 by 9 matrices. Here $\diamond$ stands for 5 by 5 matrices, + stands for 7 by 7 matrices, $\square$ stands for 9 by 9 matrices .

Table 2: Variation of Hartree Fock energy, centre of mass kinetic energy, mass and magnetic moment of $\Delta^{++}$with asymptotic parameter $\Lambda$ where $\Lambda^{\prime}$ is $350 \mathrm{MeV} ; b^{\prime}$ is $0.60, b$ is 0.84 using seven by seven matrix.

\begin{tabular}{|c|c|c|c|c|c|c|}
\hline$\Lambda(\mathrm{MeV})$ & $E_{H F}(\mathrm{MeV})$ & $T_{C M}(\mathrm{MeV})$ & $\mathrm{M}(\mathrm{MeV})$ & $\underline{\mu}_{\mu_{0}}$ & $r_{a v}(\mathrm{fm})$ & $N$ \\
\hline 100 & 1391 & 141 & 1250 & 5.77 & 1.11 & 1.000001 \\
\hline 250 & 1340 & 141 & 1199 & 5.95 & 1.13 & 1.000001 \\
\hline 300 & 1333 & 140 & 1193 & 5.99 & 1.14 & 1.000000 \\
\hline 325 & 1330 & 140 & 1190 & 6.00 & 1.14 & 1.000000 \\
\hline 375 & 1325 & 140 & 1185 & 6.02 & 1.14 & 1.000000 \\
\hline
\end{tabular}


Table 3: Variation of Hartree Fock energy, centre of mass kinetic energy, mass and magnetic moment of $\Delta^{++}$with confinement parameter $\Lambda^{\prime}$ where $\Lambda$ is $350 \mathrm{MeV} ; b^{\prime}$ is $0.60, b$ is 0.84 using seven by seven matrix.

\begin{tabular}{|c|c|c|c|c|c|c|}
\hline$\Lambda^{\prime}(\mathrm{MeV})$ & $E_{H F}(\mathrm{MeV})$ & $T_{C M}(\mathrm{MeV})$ & $\mathrm{M}(\mathrm{MeV})$ & $\frac{\mu}{\mu}_{0}$ & $r_{a v}(\mathrm{fm})$ & $N$ \\
\hline 250 & 1003 & 140 & 863 & 6.03 & 1.39 & 0.999999 \\
\hline 300 & 1171 & 139 & 1032 & 6.09 & 1.22 & 1.000001 \\
\hline 325 & 1251 & 140 & 1111 & 6.03 & 1.17 & 1.000001 \\
\hline 375 & 1399 & 140 & 1259 & 6.16 & 1.14 & 1.000000 \\
\hline 400 & 1475 & 142 & 1333 & 6.76 & 1.26 & 1.000000 \\
\hline
\end{tabular}

Table 4: Hartree Fock energy, centre of mass kinetic energy, mass and magnetic moment of $\Delta^{++}$where $\Lambda^{\prime}$ is $350 \mathrm{MeV}, \Lambda$ is $100 \mathrm{MeV}$; $b^{\prime}$ is $0.60, b$ is 0.84 using nine by nine matrix.

\begin{tabular}{|c|c|c|c|c|c|}
\hline$E_{H F}(\mathrm{MeV})$ & $T_{C M}(\mathrm{MeV})$ & $\mathrm{M}(\mathrm{MeV})$ & $\frac{\mu}{\mu}_{0}$ & $r_{a v}(\mathrm{fm})$ & $N$ \\
\hline 1354 & 130 & 1224 & 6.15 & 1.22 & 0.998476 \\
\hline
\end{tabular}

Figures (2) and (3) show the wave functions and the single particle potential for $\Lambda^{\prime}=$ $350 \mathrm{MeV}$ but $\Lambda=350 \mathrm{MeV}$ and $100 \mathrm{MeV}$. As expected, there is no change in large $\mathrm{r}$ but an insignificant variation in small $\mathrm{r}$. This is reflected in $E_{H F}$ and $\mu$ ( table 2).

On the other hand, $\Lambda^{\prime}$ variation ( figures 4 and 5) is quite significant and larger in large $\mathrm{r}$. This is reflected in $E_{H F}$ and $\mu$ ( table 3).

So we can conclude that for finite system calculations, the value of the confinement parameter $\Lambda^{\prime}$ plays a more important role in the Richardson Potential. 


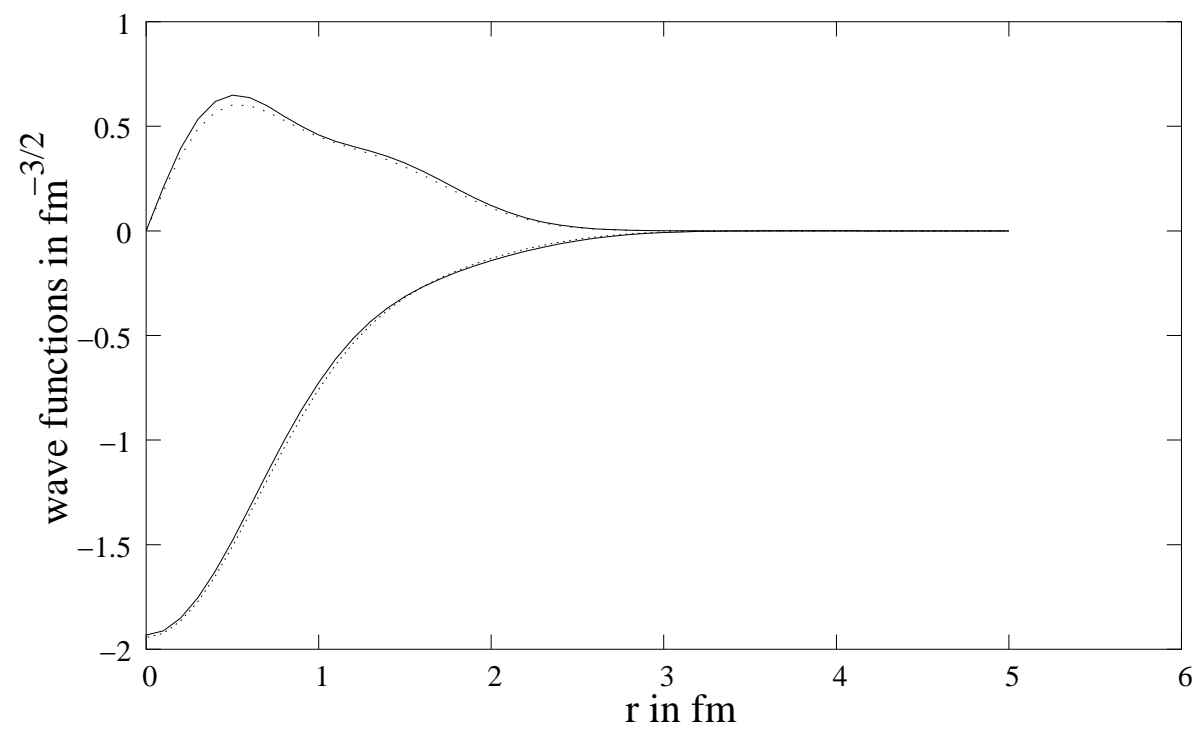

Figure 2: Variation of the wave functions with $\mathrm{r}$ for two different values of the asymptotic freedom parameter $\Lambda$ ( using seven by seven matrices ); the upper ones are G(r) and the lower ones are F(r). Here the dotted curve is for $\Lambda=\Lambda^{\prime}=350 \mathrm{MeV}$ and the solid one is for $\Lambda=100, \Lambda^{\prime}=350 \mathrm{MeV}$.



Figure 3: Variation of the single particle potential with $\mathrm{r}$ for two different values of the asymptotic freedom parameter $\Lambda$ ( using seven by seven matrices ). Here the dotted curve is for $\Lambda=\Lambda^{\prime}=350 \mathrm{MeV}$ and the solid one is for $\Lambda=100, \Lambda^{\prime}=350 \mathrm{MeV}$. 


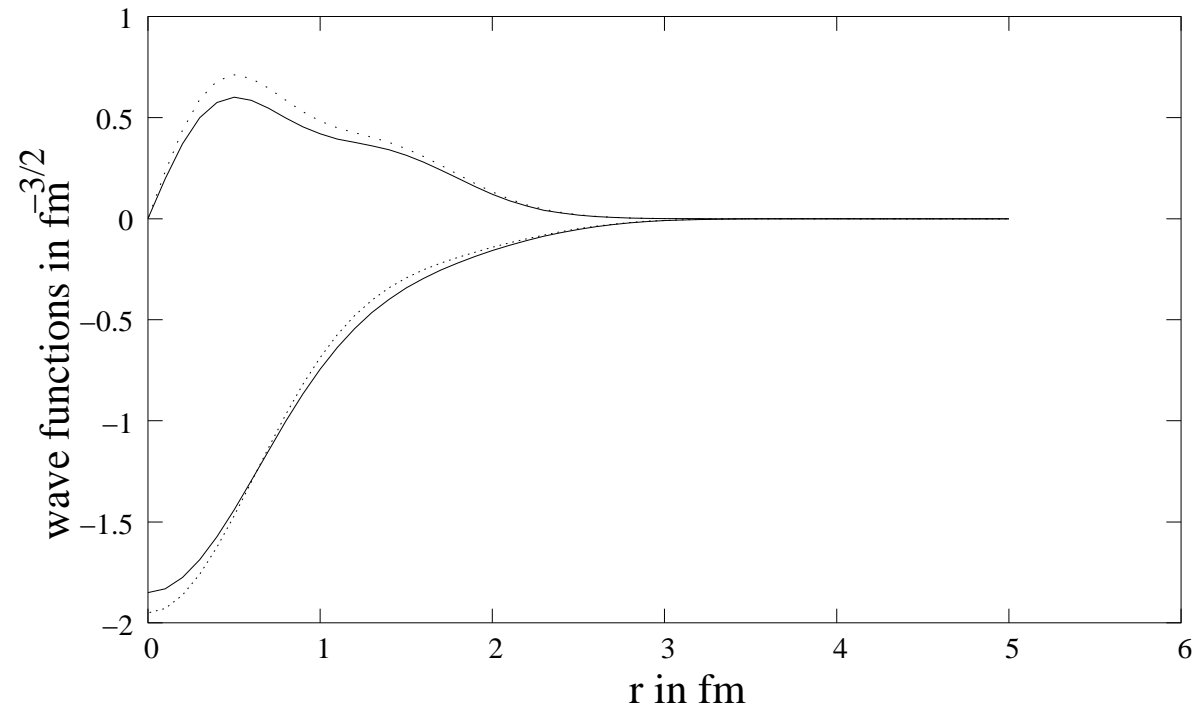

Figure 4: Variation of the wave functions with $\mathrm{r}$ for two different values of the confinement parameter $\Lambda^{\prime}$ ( using seven by seven matrices ); the upper ones are $\mathrm{G}(\mathrm{r})$ and the lower ones are $\mathrm{F}(\mathrm{r})$. Here the dotted curve is for $\Lambda^{\prime}=375 \mathrm{MeV}, \Lambda=350 \mathrm{MeV}$ and the solid one is for $\Lambda^{\prime}=325 \mathrm{MeV}, \Lambda=350 \mathrm{MeV}$.



Figure 5: Variation of the single particle potential with $\mathrm{r}$ for two different values of the confinement parameter $\Lambda^{\prime}$ ( using seven by seven matrices ). Here the dotted curve is for $\Lambda^{\prime}=375 \mathrm{MeV}, \Lambda=350 \mathrm{MeV}$ and the solid one is for $\Lambda^{\prime}=325 \mathrm{MeV}, \Lambda=350 \mathrm{MeV}$. 


\section{Result with $\Omega^{-}$}

$\Omega^{-}$is the triple s-quark state. There is no qualitative change in the procedure except for for the quark mass; $m_{s}$ is taken here as $150 \mathrm{MeV}$. Results for $\Lambda^{\prime}=\Lambda=350 \mathrm{MeV}$ is shown in table [5].

Table 5: Variation of Hartree Fock energy, centre of mass kinetic energy, mass and magnetic moment of $\Omega^{-}$with oscillator parameter $b$ where $\Lambda=\Lambda^{\prime}=350 \mathrm{MeV}, b^{\prime}$ is 0.60 .

\begin{tabular}{|c|c|c|c|c|c|c|}
\hline$b$ & $E_{H F}(\mathrm{MeV})$ & $T_{C M}(\mathrm{MeV})$ & $\mathrm{M}(\mathrm{MeV})$ & $\frac{\mu}{\mu}_{0}$ & $r_{a v}(\mathrm{fm})$ & $N$ \\
\hline 0.60 & 1639 & 176 & 1463 & -1.95 & 0.836879 & 1.000001 \\
\hline 0.62 & 1638 & 175 & 1463 & -1.95 & 0.836778 & 1.000000 \\
\hline 0.64 & 1638 & 174 & 1464 & -1.95 & 0.836879 & 1.000000 \\
\hline 0.66 & 1638 & 172 & 1466 & -1.95 & 0.836854 & 1.000001 \\
\hline 0.68 & 1638 & 171 & 1467 & -1.95 & 0.836882 & 1.000000 \\
\hline 0.70 & 1637 & 169 & 1468 & -1.95 & 0.83643 & 0.999999 \\
\hline 0.72 & 1639 & 167 & 1472 & -1.95 & 0.83695 & 1.00001 \\
\hline
\end{tabular}

We improve the fit by taking different values for $\Lambda^{\prime}$ and $\Lambda$; specifically for $\Lambda^{\prime}=350 \mathrm{MeV}$ and $\Lambda=100 \mathrm{MeV}$.

Table 6: Hartree Fock energy, centre of mass kinetic energy, mass and magnetic moment of $\Omega^{-}$ where $\Lambda^{\prime}$ is $350 \mathrm{MeV}, \Lambda$ is $100 \mathrm{MeV} ; b^{\prime}$ is $0.60, b$ is 0.70 .

\begin{tabular}{|c|c|c|c|c|c|}
\hline$E_{H F}(\mathrm{MeV})$ & $T_{C M}(\mathrm{MeV})$ & $\mathrm{M}(\mathrm{MeV})$ & $\frac{\mu}{\mu}_{0}$ & $r_{a v}(\mathrm{fm})$ & $N$ \\
\hline 1721 & 165 & 1556 & -1.92 & 0.849015 & 0.999999 \\
\hline
\end{tabular}

The centre of mass kinetic energy $T_{c m}$ is rather high resulting a lower mass of $\Omega^{-}$ 


\section{Conclusions and summary}

In summary we have shown that a RHF calculation can be done for the energies and magnetic moments of the simplest baryons $\Omega^{-}$and $\Delta^{++}$. We employed the oscillator basis with good convergence. Optimized oscillator parameters are used for the large and small components of the RHF wave functions. We belive that this model may be good base for further investigations of baryon properties. The center of mass correction is around $150 \mathrm{MeV}$ for these baryons (about $10 \%$ of $\left.E_{H F}\right)$. Quark masses chosen are 4 and $150 \mathrm{MeV}$ for $\mathrm{u}$ and s quark respectively. For q q interaction we chose Richardson potential. We separated out the confinement and asymptotic freedom scale parameter $\left(\Lambda^{\prime}\right.$ and $\left.\Lambda\right)$. From the best fit of the energies of $\Omega^{-}$and $\Delta^{++}$we find $\Lambda^{\prime}=350 \mathrm{MeV}$ and $\Lambda=100 \mathrm{MeV}$. We believe these are realistic values. Above parameters are now used in strange quark matter calculation $([21])$.

Acknowledgments :-

MB, MD, SD and JD thank IUCAA for a short pleasant stay in 2003. We acknowledge that the suggestion of doing a calculation with two $\Lambda$ came originally from Dr. Ignazio Bombaci of Pisa. We thank Monika Sinha for careful reading of the manuscript.

\section{References}

[1] G.'t Hooft, Nucl. Phys. B 72461 (1974); B 75461 (1974)

[2] "Quarks, Baryons and Chiral Symmetry", Atushi Hosaka, Hiroshi Toki ( World Scientific, 2001; page 245) and references there in.

[3] E. Witten, Nucl. Phys. B 16057 (1979)

[4] J. Dey , M Dey and J. Le. Tourneux, Phys. Rev. D 342104 (1986)

[5] J. L. Richardson, Phys. Lett. B 82272 (1979).

[6] M. Dey, I. Bombaci, J. Dey, S. Ray and B. C. Samanta, Phys. Lett. B 438 (1998) 123; Addendum B 447 (1999) 352; Erratum B 467 (1999) 303; Indian J. Phys. 73 B (1999) 377.

[7] X. Li, I. Bombaci, M. Dey, J. Dey \& E. P. J. van den Heuvel, Phys. Rev. Lett. 83 (1999) 3776 .

[8] X. Li, S. Ray, J. Dey, M. Dey \& I. Bombaci, Ap. J. 527 (1999) L51

[9] D. Gondek-Rosińska, T. Bulik, L. Zdunik, E. Gourgoulhon, S. Ray, J, Dey and M. Dey, Astronomy \& Astrophysics 3631005 (2000), astro-ph/0007004.

[10] I. Bombaci, A. V. Thampan and B. Datta, Ap. J. 541 L71, (2000).

[11] I. Bombaci and B. Datta, Ap. J. 530 L69, (2000) 
[12] S. Ray, J. Dey, M. Dey, K. Ray and B. C. Samanta, "Entropy and EOS for hot bare strange stars", Astronomy \& Astrophysics Letters 364 (2000) L89-92.

[13] M. Sinha, M. Dey, S. Ray and J. Dey, Mon. Not. R. Astron. Soc. 337 (2002) 1368-1372.

[14] Monika Sinha, Jishnu Dey, Mira Dey, Subharthi Ray and Siddhartha Bhowmick, Mod. Phys. Lett. A 17 (2002) 1783-95.

[15] Rachid Ouyed, Jishnu Dey and Mira Dey, Quark Nova, astro-ph/0105109v3, Astron. and Astrophys. Lett. 390 (2002) L39-42.

[16] R. Sharma, S. Mukherjee, Mira Dey and Jishnu Dey, Mod. Phys. Lett. A 17 (2002) 827.

[17] Monika Sinha, Jishnu Dey, Mira Dey, Subharthi Ray and Siddhartha Bhowmick, Mod. Phys. Lett. A 18 (2003) 661

[18] Banibrata Mukhopadhyay, Subharthi Ray, Jishnu Dey and Mira Dey, Astrophys. J. 584 (2003) L83-L86.

[19] G. Castro and A. Mariano, Phys. Lett. B 517 (2002) 339.

[20] M. Sinha, A.Iqubal, J. Dey and M. Dey, Phys. Lett. B 563 (2003)63.

[21] Manjari Bagchi, Ph. D. thesis.

[22] M. A. Shifman et al., Nucl. Phys. B 147385 (1979)

[23] A. E. Radzhabov and M. K. Volkov hep-ph/0305272 v1 (May 23, 2003)

[24] H. W. Crater and P. Van Alstine, Phys. Rev. Lett. 531527 (1984).

[25] "Models of the Nucleon From Quarks to Soliton ", R. K. Bhaduri (Addison Wesley, Redwood City and Sarat Book House, Kolkata, 2002). 